\title{
SYMMETRIC DUAL MULTIOBJECTIVE FRACTIONAL PROGRAMMING
}

\author{
T. WEIR
}

(Received 8 May 1989; Revised 16 October 1989)

Communicated by B. Mond

\begin{abstract}
A pair of symmetric dual multiobjective fractional programming problems is formulated and appropriate duality theorems are established.

1980 Mathematics subject classification (Amer. Math. Soc.) (1985 Revision): 90 C 31, 90 C 32.
\end{abstract}

\section{Introduction}

Dorn [5] defined a program and its dual to be symmetric if the dual of the dual is the original problem. Dantzig, Eisenberg and Cottle [4] and Mond [7] gave symmetric dual theorems for programs involving a scalar functions $f(x, y), x \in \mathbb{R}^{n}, y \in \mathbb{R}^{m}$ under the condition that $f(\cdot, y)$ is convex and $f(x, \cdot)$ is concave. More recently, Mond and Weir [8] have given a different pair of symmetric dual nonlinear programs which allows for a weakening of the convexity hypothesis for $f(x, y)$. Chandra, Craven and Mond [2] formulated a pair of symmetric dual fractional programs under suitable convexity hypothesis.

In [10] Weir and Mond discuss symmetric duality in multiobjective programming, generalizing [4] and [8]. The duals given there reduce to those known for scalar valued symmetric programming and also some more recent results in multiobjective programming duality.

This work was done while the author was an Honorary Visiting Fellow, Department of Mathematics, Australian Defence Force Academy, Campbell, ACT, 2600, Australia.

(C) 1991 Australian Mathematical Society 0263-6115/91 \$A2.00+0.00 
The purpose of this paper is to formulate a pair of symmetric dual multiobjective fractional programs under suitable convexity assumptions. The relationship of the symmetric dual programs presented here to dual, nonsymmetric, fractional programming problems is also discussed.

\section{Notation and preliminaries}

The following conventions for vectors in $\mathbb{R}^{n}$ will be used:

$x>y$ if and only if $x_{i}>y_{i}, i=1,2, \ldots, n$;

$x \geqq y$ if and only if $x_{i} \geqq y_{i}, i=1,2, \ldots, n$;

$x \geq y$ if and only if $x_{i} \geqq y_{i}, i=1,2, \ldots, n$, but $x \neq y, n \geq 2$;

$x \nsucceq y$ is the negation of $x \geq y$.

If $F$ is a twice differentiable functions from $\mathbb{R}^{n} \times \mathbb{R}^{m}$ to $\mathbb{R}$, then $\nabla_{x} F$ and $\nabla_{y} F$ denote gradient (column) vectors of $F$ with respect to $x$ and $y$ respectively, and $\nabla_{y y} F$ and $\nabla_{y x} F$ denote respectively the $(m \times m)$ and $(n \times m)$ matrices of second partial derivatives.

If $F$ is a twice differentiable function from $\mathbb{R}^{n} \times \mathbb{R}^{m} \rightarrow \mathbb{R}^{k}$, then $\nabla_{x} F$ and $\nabla_{y} F$ denote respectively the $(n \times k)$ and $(m \times k)$ matrices of first partial derivatives. Consider the multiobjective programming problem:

$$
\text { minimize } f(x) \text { subject to } x \in X \text {. }
$$

Here $f: \mathbb{R}^{n} \rightarrow \mathbb{R}^{k}$ and $X \subset \mathbb{R}^{n}$. A feasible point $z$ is said to be an efficient solution of $(\mathrm{P})$ if $f_{i}(z) \geq f_{i}(x)$ for all $i=1,2, \ldots, k$ implies $f_{i}(z)=f_{i}(x)$ for all $i=1,2, \ldots, k$.

A feasible point $z$ is said to be properly efficient [6] if it is efficient for (P) and if there exists a scalar $M>0$ such that, for each $i$,

$$
f_{i}(z)-f_{i}(x) \leq M\left(f_{j}(x)-f_{j}(z)\right)
$$

for some $j$ such that $f_{j}(x)>f_{j}(z)$ wherever $x$ is feasible for $(\mathrm{P})$ and $f_{i}(x)<f_{i}(z)$.

A feasible point $z$ is said to be a weak minimum [1] if there exists no other feasible point $x$ for which $f(z)>f(x)$. If a feasible point $z$ is efficient then it is also a weak minimum.

\section{Duality}

Consider the following pair of multiobjective symmetric fractional programs: 


\section{Primal (FP)}

minimize

$$
\left(\frac{n_{1}(x, y)}{d_{1}(x, y)}, \ldots, \frac{n_{k}(x, y)}{d_{k}(x, y)}\right)^{t}
$$

subject to

$$
\begin{gathered}
\sum_{i=1}^{k} \omega_{i}\left(d_{i} \nabla_{y} n_{i}-n_{i} \nabla_{y} d_{i}\right) \leqq 0 \\
y^{t} \sum_{i=1}^{k} \omega_{i}\left(d_{i} \nabla_{y} n_{i}-n_{i} \nabla_{y} d_{i}\right) \geq 0, \quad \omega>0, \omega^{t} e=1, \quad x \geqq 0 .
\end{gathered}
$$

Dual (FD)

maximize

$$
\left(\frac{n_{1}(u, v)}{d_{1}(u, v)}, \ldots, \frac{n_{k}(u, v)}{d_{k}(u, v)}\right)^{t}
$$

subject to

$$
\begin{gathered}
\sum_{i=1}^{k} \omega_{i}\left(d_{i} \nabla_{x} n_{i}-n_{i} \nabla_{x} d_{i}\right) \geqq 0, \\
u^{t} \sum_{i=1}^{k} \omega_{i}\left(d_{i} \nabla_{x} n_{i}-n_{i} \nabla_{x} d_{i}\right) \leq 0, \quad \omega>0, \omega^{t} e=1, v \geqq 0 .
\end{gathered}
$$

Here $e=(1,1, \ldots, 1)^{t} \in \mathbb{R}^{k} ; n_{i}, i=1,2, \ldots, k$, and $d_{i}, i=1,2, \ldots$, $k$, are twice differentiable functions from $\mathbb{R}^{n} \times \mathbb{R}^{m}$ to $R, n_{1}(\cdot, y)$ and $d_{i}(x, \cdot), i=1,2, \ldots, k$, are convex $n_{i}(x, \cdot)$ and $d_{i}(\cdot, y), i=1,2, \ldots$, $k$, are concave. It is assumed throughout that in the feasible regions $n_{i}>0$, $i=1,2, \ldots, k$, and that each $d_{i}$ is bounded.

In order to simplify notation we rewrite the primal and dual programs as follows.

Primal (FP')

minimize

$$
q=\left(q_{1}, q_{2}, \ldots, q_{k}\right)^{t}
$$

subject to

$$
\begin{gathered}
q_{i}=n_{i}(x, y) / d_{i}(x, y), \quad i=1,2, \ldots, k, \\
\sum_{i=1}^{k} \omega_{i}\left(\nabla_{y} n_{i}-q_{i} \nabla_{y} d_{i}\right) \leqq 0, \\
y^{t} \sum_{i=1}^{k} \omega_{i}\left(\nabla_{y} n_{i}-q_{i} \nabla_{y} d_{i}\right) \geq 0,
\end{gathered}
$$




$$
\omega>0, \omega^{t} e=1, x \geqq 0 .
$$

Dual $\left(\mathrm{FD}^{\prime}\right)$

maximize

$$
p=\left(p_{1}, p_{2}, \ldots, p_{k}\right)^{t}
$$

subject to

$$
\begin{gathered}
p_{i}=n_{i}(u, v) / d_{i}(u, v), \quad i=1,2, \ldots, k, \\
\sum_{i=1}^{k} \omega_{i}\left(\nabla_{x} n_{i}-p_{i} \nabla_{x} d_{i}\right) \geqq 0 \\
u^{t} \sum_{i=1}^{k} \omega_{i}\left(\nabla_{x} n_{i}-p_{i} \nabla_{x} d_{i}\right) \leq 0, \quad \omega>0, \omega^{t} e=1, v \geqq 0 .
\end{gathered}
$$

The following weak and strong duality theorems are stated in terms of $\left(\mathrm{FP}^{\prime}\right)$ and $\left(\mathrm{FP}^{\prime}\right)$ but apply equally to (FP) and (FD).

THEOREM 1 (weak duality). Let $(x, y, w)$ be feasible for (FP') and let $(u, v, w)$ be feasible for $\left(\mathbf{F P}^{\prime}\right)$. Then $q \$ p$.

Proof. From (4) and (7)

$$
(x-u)^{t}\left[\sum_{i=1}^{k} \omega_{i}\left(n_{i k}(u, v)-p_{i} d_{i k}(u, v)\right)\right] \geq 0 .
$$

The convexity and concavity assumptions imply that $n_{i}(\cdot, v)-p_{i} d_{i}(\cdot, v)$, $i=1,2, \ldots, k$, are convex; thus

$$
\sum_{i=1}^{k} \omega_{i}\left(n_{i}(x, v)-p_{i} d_{i}(x, v)\right) \geq \sum_{i=1}^{k} \omega_{i}\left(n_{i}(u, v)-p_{i} d_{i}(u, v)\right)
$$

and from (5)

$$
\sum_{i=1}^{k} \omega_{i}\left(n_{i}(u, v)-p_{i} d_{i}(u, v)\right) \geq 0
$$

From (3) and (8)

$$
(v-y)^{t}\left[\sum_{i=1}^{k} \omega_{i}\left(n_{i y}(x, y)-q_{i} d_{i y}(x, y)\right)\right] \leq 0 .
$$


The convexity and concavity assumptions imply $n_{i}(x, \cdot)-q_{i} d_{i}(x, \cdot), i=$ $1,2, \ldots, k$, are concave; thus

$$
\sum_{i=1}^{k} \omega_{i}\left(n_{i}(x, v)-q_{i} d_{i}(x, v)\right) \leq \sum_{i=1}^{k} \omega_{i}\left(n_{i}(x, y)-q_{i} d_{i}(x, y)\right)
$$

and from (1)

$$
\sum_{i=1}^{k} \omega_{i}\left(n_{i}(x, v)-q_{i} d_{i}(x, v)\right) \leq 0
$$

Combining (9) and (10) gives

$$
\sum_{i=1}^{k} \omega_{i}\left(q_{i}-p_{i}\right) d_{i}(x, v) \geq 0
$$

If, for some $i, q_{i}>p_{i}$ and for all $j \neq i, q_{j} \leq p_{j}$, then since $d_{i}>0$, $i=1,2, \ldots, k$, one would obtain a contradiction to (11); hence $q \Varangle p$.

THEOREM 2 (strong duality). Let $\left(x_{0}, y_{0}, w_{0}\right)$ be a properly efficient solution for (FP'); fix $w=w_{0}$ in (FD); define $q_{0}$ by $q_{0 i}=n_{i}\left(x_{0}, y_{0}\right) / d_{i}\left(x_{0}, y_{0}\right)$, $i=1,2, \ldots, k$. Assume that

$$
\sum_{i=1}^{k} \omega_{0 i}\left(\nabla_{y y} n_{i}\left(x_{0}, y_{0}\right)-q_{0 i} \nabla_{y y} d_{i}\left(x_{0}, y_{0}\right)\right)
$$

is positive or negative definite and that the set

$$
\left\{\left(\nabla_{y} n_{1}-q_{01} \nabla_{y} d_{1}\right),\left(\nabla_{y} n_{2}-q_{02} \nabla_{y} d_{2}\right), \ldots,\left(\nabla_{y} n_{k}-q_{0 k} \nabla_{y} d_{k}\right)\right\}
$$

is linearly independent. Then $\left(x_{0}, y_{0}, \omega_{0}\right)$ is a properly efficient solution of $\left(\mathrm{FD}^{\prime}\right)$.

Proof. Since $\left(x_{0}, y_{0}, w_{0}\right)$ is a properly efficient solution of $\left(\mathrm{FP}^{\prime}\right)$ then it is also a weak minimum. Hence there exist $a \in \mathbb{R}^{m}, b \in \mathbb{R}^{k} \quad r \in \mathbb{R}^{m}$, $s \in \mathbb{R}, t \in \mathbb{R}^{k}, z \in \mathbb{R}^{n}$, such that the following Fritz John conditions are 
satisfied at $\left(x_{0}, y_{0}, \omega_{0}\right)[3]$ :

$$
a_{i}+b_{i} d_{i}-\omega_{0 i}\left(\nabla_{y} d_{i}\right)^{t}\left(r-s y_{0}\right)=0, \quad i=1,2, \ldots, k,
$$

$$
\sum_{i=1}^{k}\left[b_{i}\left(\nabla_{x} n_{i}-q_{0 i} \nabla_{x} d_{i}\right)+\omega_{0 i}\left(\nabla_{y x} n_{i}-q_{0 i} \nabla_{y x} d_{i}\right)\left(r-s y_{0}\right)\right]-z=0,
$$

$$
\sum_{i=1}^{k}\left[\left(b_{i}-s \omega_{0 i}\right)\left(\nabla_{y} n_{i}-q_{0 i} \nabla_{y} d_{i}\right)+\omega_{0 i}\left(\nabla_{y y} n_{i}-\omega_{0 i} \nabla_{y y} d_{i}\right)\left(r-s y_{0}\right)\right]=0
$$

(17) $\quad\left(r-s y_{0}\right)^{t}\left(\nabla_{y} n_{k}-q_{0 i} \nabla_{y} d_{i}\right)-t_{i}=0, \quad i=1,2, \ldots, k$,

$$
\begin{gathered}
t^{t} \omega_{0}=0, \\
z^{t} x_{0}=0, \\
(a, r, s, t, z) \geqq 0, \\
(a, b, r, s, t, z) \neq 0 .
\end{gathered}
$$

Since $\omega_{0}>0$ and $t \geqq 0$, then $t=0$.

Multiplying (16) by $\left(r-s y_{0}\right)^{t}$ and applying (17) gives

$$
\left(r-s y_{0}\right)^{t}\left[\sum_{i=1}^{k} \omega_{0 i}\left(\nabla_{y y} n_{i}-q_{0 i} \nabla_{y y} d_{i}\right)\right]\left(r-s y_{0}\right)=0 \text {. }
$$

Since (12) is assumed positive or negative definite then

$$
r=s y_{0} \text {. }
$$

Thus, from (16),

$$
\sum_{i=1}^{k}\left(b_{i}-s \omega_{0 i}\right)\left(\nabla_{y} n_{i}-q_{0 i} \nabla_{y} d_{i}\right)=0
$$

and since, by assumption, the set (13) is linearly independent then

$$
b=s \omega_{0} .
$$

If $s=0$, then $b=0$; from (14), $a=0$; from (22) $r=0$; from (15) $z=0$; this combined with $t=0$ contradicts (21); hence $s>0$ and $b>0$. From (22), $y_{0} \geqq 0$ and from (15) and (23)

$$
\sum_{i=1}^{k} \omega_{0 i}\left(\nabla_{x} n_{i}-q_{0 i} \nabla_{x} d_{i}\right) \geqq 0
$$

From (15), (23) and (19) it also follows that

$$
x_{0}^{t} \sum_{i=1}^{k} \omega_{0 i}\left(\nabla_{x} n_{i}-q_{0 i} \nabla_{x} d_{i}\right)=0
$$


Thus, $\left(x_{0}, y_{0}, \omega_{0}\right)$ is feasible for (FD) and the objective values of (FP') and $\left(\mathrm{FD}^{\prime}\right)$ are equal there. Clearly, $\left(x_{0}, y_{0}, \omega_{0}\right)$ is efficient for $\left(\mathrm{FD}^{\prime}\right)$. If $\left(x_{0}, y_{0}, \omega_{0}\right)$ were improperly efficient, then for some feasible $\left(u_{i}, v_{i}, \omega_{0}\right)$ with $p_{1 i}=n_{i}\left(u_{i}, v_{i}\right) / d_{i}\left(u_{i}, v_{i}\right), i=1,2, \ldots, k$, and for some $i, p_{1 i}-$ $q_{0 i}>M$ for any $M>0$. Since $d_{i}, i=1,2, \ldots, k$, is bounded it follows that

$$
\sum_{i=1}^{k} \omega_{0 i}\left(q_{0 i}-p_{1 i}\right) d_{i}\left(x_{0}, v_{i}\right)<0,
$$

which contradicts weak duality, equation (ii). Thus $\left(x_{0}, y_{0}, \omega_{0}\right)$ is properly efficient for $\left(\mathrm{FD}^{\prime}\right)$.

\section{Special cases}

(i) If $n_{i}(x, y)=f_{i}(x)+y^{t} h(x), i=1,2, \ldots, k$, and $d_{i}(x, y)=g_{i}(x)$, $i=1,2, \ldots, k$, where $f_{i}, g_{i}: \mathbb{R}^{n} \rightarrow \mathbb{R}, i=1,2, \ldots, k$, and $h: \mathbb{R}^{n} \rightarrow \mathbb{R}^{m}$ then programs (FP) and (FD) reduce to

$$
\text { minimize }\left(\left(f_{1}(x)+y^{t} h(x)\right) / g_{1}(x), \ldots,\left(f_{k}(x)+y^{t} h(x)\right) / g_{k}(x)\right)^{t}
$$

subject to

$$
h(x) \leqq 0, \quad y^{t} h(x) \geq 0, \quad x \geqq 0,
$$

and

(D1) maximize $\left(\left(f_{1}(u)+v^{t} h(u)\right) / g_{1}(u), \ldots,\left(f_{k}(u)+v^{t} h(u)\right) / g_{k}(u)\right)^{t}$ subject to

$$
\begin{gathered}
\sum_{i=1}^{k} \omega_{i} g_{i}(u) \nabla\left(\left(f_{i}(u)+v^{t} h(u)\right) / g_{i}(u)\right) \geqq 0, \\
u^{t} \sum_{i=1}^{k} \omega_{i} g_{i}(u) \nabla\left(\left(f_{i}(u)+v^{t} h(u)\right) / g_{i}(u)\right) \leq 0, \\
\omega>0, \quad \omega^{t} e=1, \quad v \geqq 0 .
\end{gathered}
$$

$\left(\right.$ Here $\nabla \equiv \nabla_{x}$.)

Since in $(\mathrm{P} 1) y^{t} h(x) \geq 0, g_{i}(x)>0, i=1,2, \ldots, k$, we can take $y=0$ and thus eliminate $y$ from the problem. The problem (P1) is thus equivalent to

$$
\operatorname{minimize}\left(f_{1}(x) / g_{1}(x), f_{2}(x) / g_{2}(x), \ldots, f_{k}(x) / g_{k}(x)\right)^{t}
$$

subject to

$$
h(x) \leqq 0, \quad x \geqq 0
$$


This is a standard multiobjective fractional programming problem, with nonnegativity constraints. Program (D1) is a Mond-Weir type dual for (P2).

(ii) If, in (FP) and (FD), $d_{i}(x, y)=1$, we obtain symmetric dual problems of Weir and Mond [10]; there duality is proved under somewhat weaker convexity conditions.

(iii) If, in (FP) and (FD), $k=1$, then we obtain pair of scalar symmetric dual fractional programs of Chandra, Craven and Mond [2].

\section{References}

[1] J. M. Borwein, Optimization with respect to partial orderings, (D. Phil., Thesis, Oxford University, 1974).

[2] S. Chandra, B. D. Craven and B. Mond, 'Symmetric dual fractional programming', Z. Oper. Res. 29 (1985), 59-64.

[3] B. D. Craven, 'Lagrangian conditions and quasiduality', Bull. Austral. Math. Soc. 16 (1977), 325-339.

[4] G. G. Dantzig, E. Eisenberg and R. W. Cottle, 'Symmetric dual nonlinear programs', Pacific J. Math. 15 (1965), 809-812.

[5] W. S. Dorn, 'A symmetric dual theorem for quadratic programs', J. Oper. Res. Soc. Japan 2 (1960), 93-97.

[6] A. M. Geoffrion, 'Proper efficiency and the theory of vector maximization', J. Math. Anal. Appl. 22 (1968), 613-630.

[7] B. Mond, 'A symmetric dual theorem for nonlinear programming', Quart. Appl. Math. 23 (1965), 265-269.

[8] B. Mond and T. Weir, 'Generalized concavity and duality', in Generalized Concavity in Optimization and Economics, edited by S. Schaible and W. T. Ziemba, pp. 263-279, (Academic Press, New York, 1981).

[9] T. Weir, 'Proper efficiency and duality for vector valued optimization problems', J. Austral. Math. Soc. Ser. A 43 (1987), 21-35.

[10] T. Weir and B. Mond, 'Symmetric and self duality in multiple objective programming', Asia Pacific J. Oper. Res. 5 (1988), 124-133.

\section{Boehm Close}

Isaacs

ACT 2607

Australia 